\title{
Plasma convection across the polar cap, plasma mantle and cusp: Cluster EDI observations
}

\author{
H. Vaith ${ }^{1}$, G. Paschmann ${ }^{1}$, J. M. Quinn ${ }^{2}$, M. Förster ${ }^{1}$, E. Georgescu ${ }^{1}$, S. E. Haaland ${ }^{1}$, B. Klecker ${ }^{1}$, C. A. Kletzing ${ }^{3}$, \\ P. A. Puhl-Quinn ${ }^{1,2}$, H. Rème ${ }^{4}$, and R. B. Torbert ${ }^{2}$ \\ ${ }^{1}$ Max-Planck-Institut für extraterrestrische Physik, 85748 Garching, Germany \\ ${ }^{2}$ University of New Hampshire, Durham, NH 03824, USA \\ ${ }^{3}$ University of Iowa, Iowa City, IA 52242, USA \\ ${ }^{4}$ CESR-CNRS, 31028 Toulouse, France
}

Received: 2 October 2003 - Revised: 16 March 2004 - Accepted: 14 April 2004 - Published: 14 July 2004

Part of Special Issue "Spatio-temporal analysis and multipoint measurements in space"

\begin{abstract}
In this paper we report measurements of the polar cap convection obtained with the Electron Drift Instrument (EDI) on Cluster. We use 20 passes that cross the polar cap between its dayside and nightside boundaries (or vice versa) at geocentric distances ranging from about 5 to about $13 R_{E}$, and at interspacecraft separations (transverse to the ambient magnetic field) between a few $\mathrm{km}$ and almost $10000 \mathrm{~km}$. We first illustrate the nature of the data by presenting four passes in detail. They demonstrate that the sense of convection (anti-sunward vs. sunward) essentially agrees with the expectations based on magnetic reconnection occurring on the dayside or poleward of the cusp. The most striking feature in the EDI data is the occurrence of largeamplitude fluctuations that are superimposed on the average velocities. One type of fluctuation appears to grow when approaching the dayside polar cap boundary. The examples also show that there is a variable degree of inter-spacecraft correlation, ranging from excellent to poor. We then present statistical results on all 20 passes. Plotting 10-min averages of the convection velocities vs. IMF $B_{z}$ one recovers the expected dependence, albeit with large scatter. Looking at the variances computed over the same 10-min intervals, one confirms that there is indeed one type of contribution that grows towards the dayside boundary, but that variances can be high anywhere. Finally, computing the inter-spacecraft correlations as a function of their separation distance transverse to the magnetic field shows that the average correlation drops with increasing distance, but that even at distances as large as $5000 \mathrm{~km}$ the correlation can be very good. To put those scales into context, the separation distances have also been scaled to ionospheric altitudes where they range between a few hundred meters and $600 \mathrm{~km}$.
\end{abstract}

Key words. Magnetospheric physics (plasma convection; instruments and techniques; magnetopause, cusp and boundary layers)

\section{Introduction}

Magnetospheric convection is primarily driven by magnetic reconnection. For southward IMF, reconnection occurs along an X-line on the dayside magnetopause. Once interconnected, open magnetic flux tubes are carried by the solar wind over the poles downstream, penetrating deeper and deeper into the magnetotail (where they eventually will reconnect again): anti-sunward convection of magnetic flux over the polar caps is the result. The solar wind plasma moving along these field lines fills a region, the plasma mantle, that is ever widening as a result of the superposition of fieldaligned motion and inward convection.

For northward IMF, reconnection can occur poleward of the cusps, between interplanetary magnetic field lines and open tail-lobe field lines (see Lockwood and Moen, 1999, and references therein). Those lobe field lines then move toward the X-line located behind the cusps. After reconnection, one segment of these lobe field lines is disconnected from the Earth and stripped off the top of the tail lobe, while the other segment is pulled to the flanks of the magnetosphere. The result is a circulation pattern, often described as tail lobe stirring, with sunward convection (over part of the polar cap) as a necessary consequence of reconnection under northward IMF. Details of the pattern depend on IMF $B_{y}$ (Reiff and Burch, 1985). 


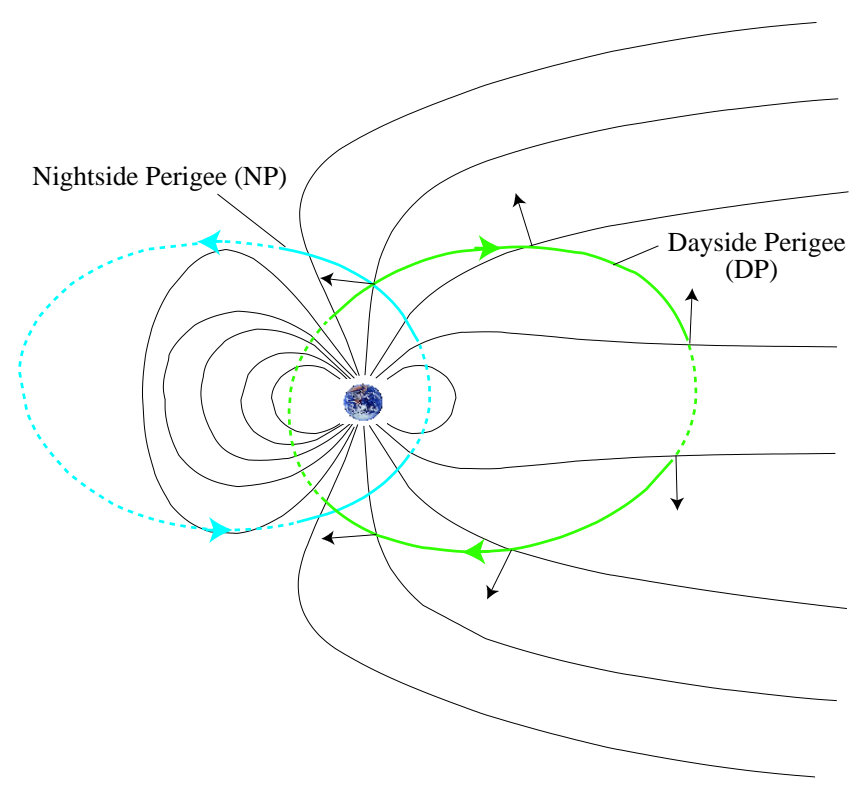

Fig. 1. The two types of orbits selected for this study, denoted Nightside Perigee (NP) and Dayside Perigee (DP), respectively. The solid parts denote the portions over the polar cap and into the polar cusps. The black arrows show the orientation of the $\hat{\boldsymbol{e}}_{s}$-axis used to define the sense of the convection in the GSE (X, Z)-plane.

Much of our knowledge of flow in the polar cap comes from ground observations with radars and magnetometers. They provide almost synoptic maps, but have limited spatial and temporal resolution. The SuperDARN radars, for example, perform a scan over 16 discrete directions every two minutes and use range gates of approximately $45 \mathrm{~km}$ along the line of sight. Preprocessing of the data yields a spatial resolution of approximately $100 \mathrm{~km}$ (Ruohoniemi and Baker, 1998). Convection measurements from low-altitude spacecraft have contributed much as well. They traverse the polar cap in about 15 min every $90 \mathrm{~min}$. By contrast, Cluster crosses the polar cap magnetic field lines at geocentric distances of $5-13 R_{E}$ (see Fig. 1), where the polar cap is much larger as a result of the divergence of the magnetic field. Coupled with the small orbital velocity at these altitudes, a polar cap traversal takes typically $4 \mathrm{~h}$ or longer. Solar wind conditions are rarely stable enough over such long times to infer, say, the polar cap potential from integration along the orbit. But, by the same token, the residence times over the polar cap are long enough to allow observations of the response to IMF changes.

The expansion of the flux tube diameters and the slow orbital motion also means that even moderate time resolution measurements translate into excellent spatial resolution when mapped into the ionosphere. Over the central polar cap the Cluster spacecraft speed is 3-4 $\mathrm{km} \mathrm{s}^{-1}$. Even a $1 \mathrm{~s}$ s-resolution measurement (as provided by EDI) at $100 \mathrm{nT}$ maps to only a few hundred meters in the ionosphere. Similarly, Cluster spacecraft separations of 100 to $10000 \mathrm{~km}$ map to very small scales in the ionosphere.
The purpose of the present paper is to report convection measurements made with the Electron Drift Instrument (EDI) over the polar cap between its dayside and nightside boundaries. Emphasis is on the dependence on the IMF orientation, the characterization of rapid temporal variations, and the correlation between the Cluster spacecraft. In Sect. 2 we briefly discuss the EDI technique and data analysis methods. Section 3 presents four polar cap passes that illustrate the nature of the observations. In Sect. 3.3 statistical results on the average convection, their fluctuations and the interspacecraft correlations are presented for a total of 20 passes. The results are discussed and summarized in Sect. 4.

\section{Instrumentation and data analysis}

The Electron Drift Instrument (EDI) measures the plasma convection velocity through the injection of artificial electron beams. The EDI technique, hardware, operation, and data analysis method have been described in detail in earlier publications (Paschmann et al., 1997; Quinn et al., 2001; Paschmann et al., 2001). Briefly, the technique is based on the injection of two electron beams at right angles to the ambient magnetic field, and the search of the two directions (within the $\boldsymbol{B}_{\perp}$-plane) that return the beams to their associated detectors after one or more gyrations. Knowledge of the positions of the guns and of the firing directions uniquely determines the drift velocity. This is the basis of the triangulation technique. Through triangulation one directly determines the "drift-step" vector $\boldsymbol{d}$, which is the displacement of the electrons after a gyro time $T_{g}$. The location in the $\boldsymbol{B}_{\perp}$-plane, from which electrons reach the detector after one gyration, can be viewed as the "target" for the electron beam.

Our standard analysis technique consists in selecting all returning beams within a certain time-interval, typically one spacecraft spin, i.e. approximately $4 \mathrm{~s}$ (but often also $1 / 2$ or $1 / 4 \mathrm{spin}$ ), and to perform an automated determination of the drift velocity. The triangulation analysis determines the drift step by searching for the target-point that minimizes an appropriate "cost-function". For each grid-point in the $\boldsymbol{B}_{\perp}$-plane, the cost-function is constructed by adding up the (squared) angle-deviations of all beams in a chosen time interval from the direction to that grid-point, normalized by the (squared) error in the firing directions. The grid-point with the smallest value of the cost-function is taken as the target. Errors in magnitude and direction are obtained from the (reduced) chi-square surface.

When the drift step becomes large, the triangulation method degenerates. Under these conditions, however, the times-of-flight of the electrons in the two beams become measurably different and can be used to determine the drift step (ToF-method). The measured times-of-flight are also used to identify the number of gyrations the emitted electrons perform prior to their detection. This information is essential for calculating the drift velocity from either the triangulation or the time-of-flight technique. 
Table 1. Key characteristics of the investigated cases.

\begin{tabular}{|c|c|c|c|c|c|c|}
\hline Case & Date & UT & Orbit & $\mathrm{C} 3-\mathrm{C} 1(\mathrm{~km})$ & IMF & $\mathrm{AE}$ \\
\hline 1 & 13/14 Feb. 2001 & 20:10-00:45 & in, $\mathrm{NP}_{A}$ & 690-799 & $\mathrm{SBz}$ & 800 \\
\hline 2 & 23 Feb. 2001 & 09:05-13:00 & in, $\mathrm{NP}_{A}$ & $720-894$ & $\mathrm{SBz}$ & 500 \\
\hline 3 & 23 Feb. 2001 & $16: 10-20: 10$ & out, $\mathrm{NP}_{A}$ & $442-490$ & $\mathrm{SBz}(\mathrm{NBz})$ & 450 \\
\hline 4 & 04 March 2001 & $20: 47-23: 55$ & in, $\mathrm{NP}_{A}$ & $696-863$ & $\mathrm{SBz}$ & 1800 \\
\hline 5 & 05 March 2001 & 06:00-08:06 & out, $\mathrm{NP}_{A}$ & $377-463$ & $\mathrm{SBz} / \mathrm{NBz}$ & 450 \\
\hline 6 & 16 March 2001 & $20: 00-23: 10$ & in, $\mathrm{NP}_{B}$ & 794-926 & $\mathrm{SBz}$ & 100 \\
\hline 7 & 17 March 2001 & 01:45-06:00 & out, $\mathrm{NP}_{B}$ & $545-480$ & $\mathrm{SBz} / \mathrm{NBz}$ & $<50$ \\
\hline 8 & 21 March 2001 & $21: 00-24: 00$ & out, $\mathrm{NP}_{B}$ & $410-468$ & $\mathrm{NBz}$ & $<50$ \\
\hline 9 & 13 Aug. 2001 & $11: 00-19: 00$ & in, DP & $4261-9208$ & Var. & 500 \\
\hline 10 & 13 Aug. 2001 & 02:00-13:00 & out, DP & $7711-2366$ & Var. & 750 \\
\hline 11 & 11 Sept. 2001 & $13: 40-16: 50$ & out, DP & $10870-6260$ & $\mathrm{SBz}$ & 450 \\
\hline 12 & 21 Sept. 2001 & $04: 00-11: 40$ & out, DP & $7952-2883$ & $\mathrm{NBz}$ & 50 \\
\hline 13 & 22/23 Sept. 2001 & $12: 00-08: 00$ & in, DP & 2096-11303 & Var. & 1300 \\
\hline 14 & 23 Sept. 2001 & $12: 30-00: 10$ & out, DP & 8878-2392 & $\mathrm{SBz}(\mathrm{NBz})$ & 1300 \\
\hline 15 & 09 Oct. 2001 & $06: 00-23: 00$ & in, DP & $2381-10400$ & $\mathrm{SBz} / \mathrm{NBz}$ & 900 \\
\hline 16 & 10 Oct. 2001 & $05: 15-13: 00$ & out, DP & $7565-2800$ & Var. & 500 \\
\hline 17 & 04 March 2002 & $07: 15-08: 50$ & out, $\mathrm{NP}_{A}$ & $171-130$ & $\mathrm{NBz}$ & 50 \\
\hline 18 & 23 March 2002 & $07: 20-10: 50$ & out, $\mathrm{NP}_{A}$ & $193-111$ & $\mathrm{NBz}$ & 50 \\
\hline 19 & 30 March 2002 & $09: 30-13: 28$ & out, $\mathrm{NP}_{A}$ & $236-120$ & Var. & 600 \\
\hline 20 & 13 April 2002 & $07: 50-13: 30$ & in, $\mathrm{NP}_{A}$ & $116-248$ & $\mathrm{SBz}(\mathrm{NBz})$ & 850 \\
\hline
\end{tabular}

To calculate the drift velocity from the times-of-flight, the beam firing directions need to be separated into two classes, one comprising beams that have a component parallel to the target direction; the other class consists of beams with anti-parallel components. The target direction is not known a priori, but found (with an ambiguity of $180^{\circ}$ ) by varying a reference direction such that the standard deviations of the distributions of beam firing directions in the two classes with components parallel and anti-parallel to the reference direction around their respective average directions are minimized. The ambiguity in the target direction is removed by calculating the average time-of-flight for each of the two classes. The class of beams with the larger average times-offlight then constitutes the beams directed towards the target (because those execute slightly more than one gyration), the other class constitute those directed away from the target (executing slightly less than one gyration). If the magnetic field had constant magnitude during the analysis interval (e.g. a spin period), then the drift magnitude (and its errors) could simply be computed from the difference between the average magnitude (and standard deviation) of the times-of-flight of the towards and away beams. But as this assumption is often not fulfilled, we use the high-resolution magnetic field data from the FGM instrument (Balogh et al., 1997), together with the best available calibration parameters, to compute the gyro time and its variation during the analysis interval. For each beam we then take the differences between the measured times-of-flight and the value of the gyro time at the time the beam was recorded. These differences are then summed separately for the towards and away sets, and the drift velocity (and its error) are computed from the difference between the two averages. The multi-gyration assignment is based on a comparison between the measured times-of-flight and the gyro-time. We use the gyro-times based on the highresolution FGM data for this purpose as well.

Convection velocities can also be derived from the EFW double probe electric field measurements (Gustafsson et al., 1997) and from the ion bulk velocities measured by the CIS instrument (Rème et al., 2001). By their very nature, the EDI measurements offer some unique advantages over these techniques, but also suffer from a number of limitations. The advantages over the double-probe technique lie in EDI's insensitivity to spacecraft-induced wake effects which can obscure the small electric fields found, for example, in the lowdensity environment over the polar caps. Moreover, EDI measures both components of the convection velocity for all orientations of the magnetic field, while the double-probe technique, unless when using a boom along the spin axis of the spacecraft, measures only the component of the electric field in the spin plane. The drift velocities computed as the perpendicular component of the CIS bulk velocities naturally suffer from poor counting statistics when densities are low. Bulk velocities are also affected by pressure gradients to which EDI is immune. On the other hand, EDI is adversely affected in three ways. First, rapid time variations (on spinperiod scale) in magnetic and/or electric fields can cause variations in the drift velocity (magnitude and direction) that are too rapid to be tracked by the electron beam pointing hardand software. Second, magnetic field magnitudes which are too low make the gyro radius of the emitted electrons so large 


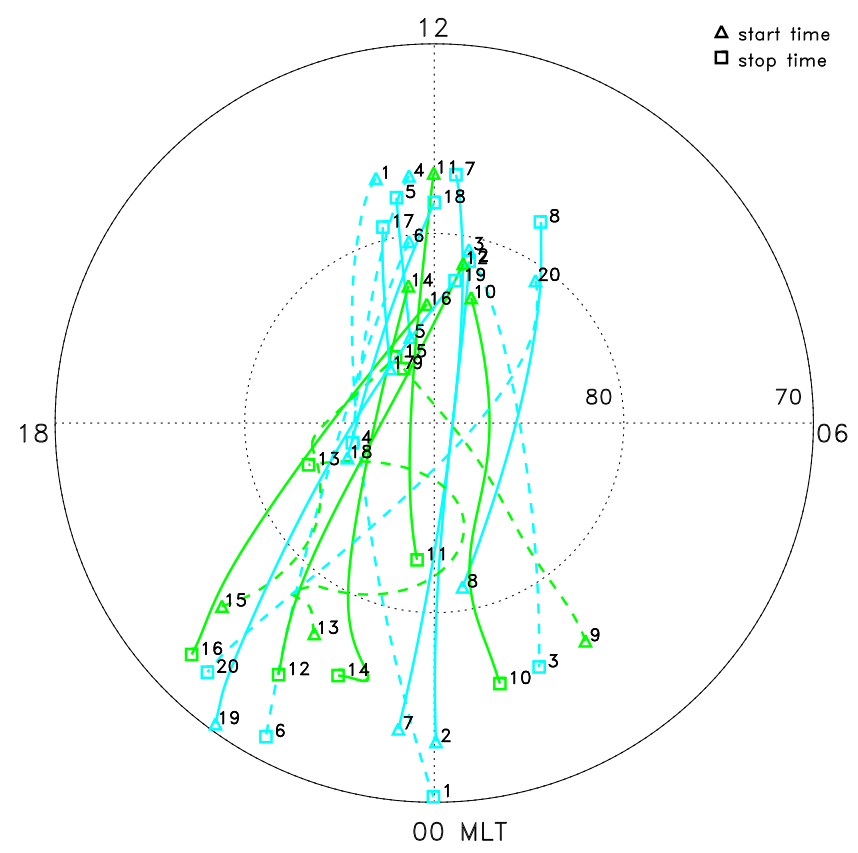

Fig. 2. Projection of the 20 passes into the polar ionosphere along the Tsyganenko-2001 model magnetic field. Solid and dashed lines are for orbits in the Northern and Southern Hemispheres, respectively. Nightside Perigee orbits (NP) are shown in blue, Dayside Perigee orbits (DP) in green, as in Fig. 1.

that the angular divergence of the beams makes the returning flux density too low to be detected. Third, the presence of ambient electrons at the beam energy cause background counts that can reduce the signal-to-noise ratio until the beams are no longer recognized.

Given the advantages and shortcomings of EDI, it is evident that the polar cap is an ideal environment for EDI because the three adverse effects just mentioned are essentially absent and the other techniques quickly reach their limitations, as demonstrated in a separate publication (Eriksson et al., submitted to Ann. Geophys., $2003^{1}$ ).

To put the EDI data into the proper context, we use the plasma densities and bulk velocities from the CIS-HIA instrument and the magnetic field magnitude measured by the FGM instrument.

\section{Observations}

\subsection{Data selection}

To characterize the convection observed with EDI over the polar cap and its dayside boundary regions (cusp and plasma mantle), we have selected a total of 20 crossings. In order to have coverage of polar cap, plasma mantle and cusp we have chosen orbits that have their plane close to the noon-midnight

\footnotetext{
${ }^{1}$ Eriksson, A. I., et al.: Electric Field Measurements on Cluster: Comparing the Double-Probe and Electron Drift Techniques, submitted to Ann. Geophys., 2003.
}

meridional plane. A further criterion for the selection of the orbits was the requirement to have continuous coverage with EDI data. Key characteristics of the selected orbits are provided in Table 1. These include the UT intervals, the separation distance between $\mathrm{C} 1$ and $\mathrm{C} 3$, an indication of the IMF orientation, and the maximum $\mathrm{AE}$ index during the crossing.

The selected orbits are categorized into two types, as illustrated in Fig. 1. "Nightside Perigee" orbits (NP) cross the nightside auroral oval into the polar cap and traverse the polar cap until they encounter the cusp or exit into the magnetosheath. "Dayside Perigee" orbits (DP) cross the northern cusp at mid-altitudes and then spend a long time in the tail lobes before entering the plasma sheet near apogee. DPorbits never cross the magnetopause. (The above description applies to outbound passes, i.e. Northern Hemisphere. The sequence is reversed for inbound passes.)

As the figure shows, the two orbit types sample the dayside and nightside boundaries of the polar cap at very different altitudes. Only near the central polar cap are they at the same altitude. Of the 20 selected passes, 12 are of type NP, 8 of type DP.

NP-orbits can conveniently be subdivided into those orbits $\left(\mathrm{NP}_{A}\right)$ that exit the polar cap directly from the lobes, i.e. poleward of the cusp or via the distant (exterior) cusp, and those $\left(\mathrm{NP}_{B}\right)$ that cross the distant cusp, but then enter the dayside magnetosphere before crossing the (dayside) magnetopause into the magnetosheath. Of the $12 \mathrm{NP}$ passes, 9 are of type $\mathrm{NP}_{A}$ and 3 of type $\mathrm{NP}_{B}$.

Figure 2 shows the 20 passes, mapped into the ionosphere along a model magnetic field (Tsyganenko, 2002). The numbers in Fig. 2 refer to the case numbers in the table.

The limitations inherent in the EDI technique noted in Sect. 2 affect the different types of passes in different ways. NP passes can suffer from a $B$ which is too low, being encountered before the magnetopause or within the distant cusp. As a consequence, the data sometimes stop before the cusp crossing was completed or the magnetopause was reached. DP passes exit the polar cap when the spacecraft enter the plasma sheet at large distances where $B$ is also often too low.

\subsection{Case studies}

In this section we will discuss four cases in some detail, in order to illustrate the character of the data. The convection velocities are presented in a coordinate system that takes into account the large change in magnetic field inclination encountered along the Cluster orbit (cf. Fig. 1) and the fact that, by its very nature, the convection velocity, $\boldsymbol{V}$, is a two-dimensional vector in the plane perpendicular to $\boldsymbol{B}$. The axes of this coordinate system are $\hat{\boldsymbol{e}}_{s}$ and $\hat{\boldsymbol{e}}_{d}$, defined as $\hat{\boldsymbol{e}}_{s}=\boldsymbol{B} \times \hat{\boldsymbol{y}}_{\mathbf{G S E}} /\left|\boldsymbol{B} \times \hat{\boldsymbol{y}}_{\mathbf{G S E}}\right|$ and $\hat{\boldsymbol{e}}_{d}=\hat{\boldsymbol{e}}_{s} \times \boldsymbol{B} / B$, respectively, and we denote the components along them as $V_{s}$ and $V_{d}$. With this definition the unit vectors $\hat{\boldsymbol{e}}_{s}, \boldsymbol{B} / B$ and $\hat{\boldsymbol{e}}_{d}$ constitute a right-hand coordinate system and a positive $V_{s}$ always maps to sunward convection in the ionosphere, even if the GSE-x component of the convection velocity becomes 


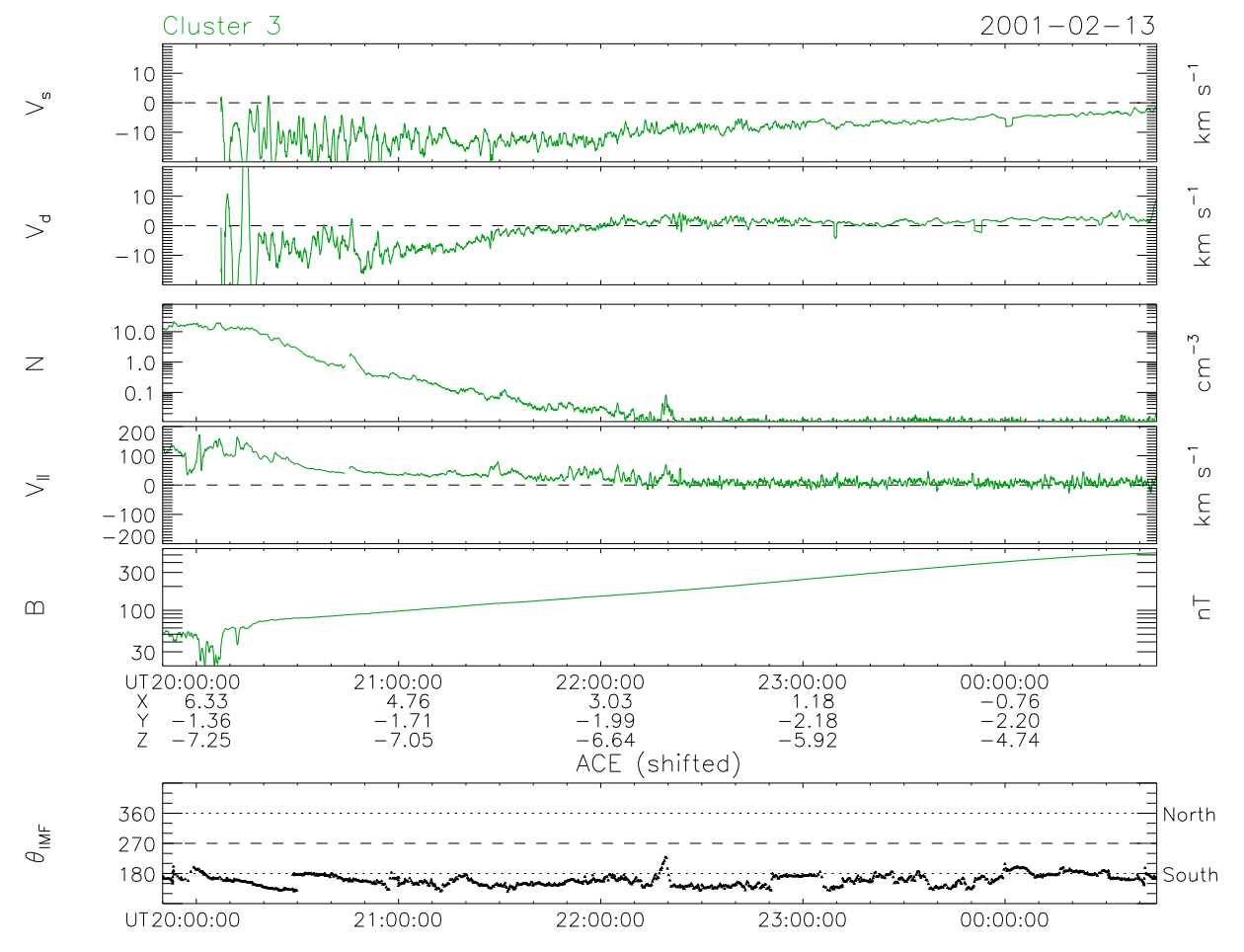

Fig. 3. Measured time-series for an inbound pass of Cluster- 3 over the southern polar cap on 13 February 2001. The top two panels show the convection velocities after applying a $30 \mathrm{~s}$-smoothing to the full-resolution EDI data; $V_{S}$ is the component along the $\hat{\boldsymbol{e}}_{s}$-axis introduced in the text, $V_{d}$ the component along $\hat{\boldsymbol{e}}_{d}$. The $3 \mathrm{rd}$ and 4th panels show the ion density, $\mathrm{N}$, and the parallel component of the bulk velocity, $V_{\|}$, measured by CIS-HIA, the 5th panel the magnetic field strength measured by FGM. Universal time, and GSE positions are shown along the bottom of the latter panel. The bottom panel finally shows the IMF clock-angle derived from the ACE magnetic field measurements, shifted for the propagation delay. Values of $0^{\circ}$ and $180^{\circ}$ denote fields that when projected into the GSM (Y, Z)-plane are pointing due north and due south, respectively.

negative where the GSE-z component of $\boldsymbol{B}$ switches sign in the magnetotail (cf. Fig. 1). The unit vector $\hat{\boldsymbol{e}}_{d}$ always has a positive GSE-y (i.e. duskward) component. In fact, for the passes selected for this study the angle between $\hat{\boldsymbol{e}}_{d}$ and $\hat{\boldsymbol{y}}_{\mathbf{G S E}}$ is on average only 16 degrees and the component of the convection velocity $\boldsymbol{V}$ along $\hat{\boldsymbol{e}}_{d}$ is thus in most cases very similar to the GSE-y component of $\boldsymbol{V}$.

\subsubsection{February 2001}

The first case, shown in Fig. 3, is an inbound pass of type $\mathrm{NP}_{A}$ that begins in the magnetosheath, encounters a low- $B$ interval that marks the magnetopause, before entering the southern tail-lobe near 20:08 UT and heading for perigee on the nightside. The IMF is directed southward throughout the time shown, as can be seen from the IMF clock angle in the bottom panel, which stays within $45^{\circ}$ of $180^{\circ}$. As this orientation favors dayside reconnection, $V_{s}$ is consistently negative, corresponding to anti-sunward convection, as expected. At the beginning of the pass the spacecraft position maps to an ionospheric position that, according to convection models (e.g. Weimer, 1996) is located near the dayside portion of the dusk convection cell. This would explain the initially negative $V_{d}$ component (at this time the angle between the $\hat{\boldsymbol{e}}_{d^{-}}$ axis and $\hat{y}_{\text {GSE }}$ is approximately five degrees and $V_{d}$ is thus very close to the GSE-y component of the convection velocity.) Also, as expected for dayside reconnection, plasma at elevated density that is flowing along $\boldsymbol{B}$ away from the Earth extends well into the tail lobe, i.e. the spacecraft has entered the plasma mantle. (Note that the CIS-CODIF instrument observes upstreaming $\mathrm{O}^{+}$ions over the polar cap on this crossing. This means that the densities shown in Fig. 3, which are calculated from the CIS-HIA measurements under the assumption that all ions are protons, are not strictly correct.)

An important feature to note is the large-amplitude fluctuations that are superimposed on the average convection velocities. These fluctuations have time scales of approximately two to five minutes and amplitudes that are comparable to the average velocities. They die out with elapsed time (or distance) from the magnetopause crossing, or when the plasma density has dropped to background values. Towards the end of the pass the velocities become very small, only $2 \mathrm{~km} \mathrm{~s}^{-1}$. If one prefers to think in terms of electric fields rather than drift velocities, one should remember that $V=1000 E / B$, with $E$ in $\mathrm{mVm}^{-1}, B$ in nT, and $V$ in $\mathrm{km} \mathrm{s}^{-1}$. The $2 \mathrm{~km} \mathrm{~s}^{-1}$ drift velocity just mentioned, which occurs at about $500 \mathrm{nT}$, thus corresponds to an electric field of $1 \mathrm{mVm}^{-1}$. 
This pass serves as a key example for the comparison between the EDI convection measurements and those from the EFW electric field and CIS bulk velocity measurements ${ }^{2}$.

\subsubsection{September 2001}

This is a DP pass 6 months later, when perigee is near local noon and apogee in the magnetotail (Fig. 4). The (outbound) pass begins with a crossing of the northern mid-altitude cusp, as evidenced by the peaks in the $\mathrm{C} 1$ and $\mathrm{C} 3$ density traces that are accompanied by brief $V_{\|}$flow pulses that are directed downward, i.e., towards the ionosphere. Consistent with their large separation $(\approx 11000 \mathrm{~km}$, see see Table 1$)$, the cusp encounters by $\mathrm{C} 1$ and $\mathrm{C} 3$ are displaced by more than $30 \mathrm{~min}$. The spacecraft then enter the plasma mantle (recognized by negative, i.e. outward directed $V_{\|}$), before entering the polar cap and heading for apogee in the magnetotail. On this day Cluster data collection stopped at 16:50 UT, well before the nightside polar cap boundary.

The IMF is along + $\mathrm{Y}$ initially, then flips to $-\mathrm{Y}$ at approximately 14:21 UT, before becoming more and more southward. The average convection is anti-sunward, with some large-amplitude fluctuations superimposed. For this pass we show EDI data for $\mathrm{C} 1, \mathrm{C} 2$ and $\mathrm{C} 3$. During the early part of the pass the spaceraft are in very different regions. As a result the velocities differ between the three spacecraft. After 15:15 UT, however, the convection becomes steady and very well correlated between the spacecraft, which at this time were more than $6000 \mathrm{~km}$ apart. This indicates a transition from a crossing of spatial structures to the encounter of large-scale temporal variations.

A noteworthy feature is the transient, sudden reductions in the convection velocity that occur later on, in particular near 16:20, when the IMF is very steady. Such dropouts are common for crossings with large $\mathrm{AE}$, and are the subject of a separate publication ${ }^{3}$ where it is demonstrated that they are associated with global magnetic field reconfigurations.

\subsubsection{March 2002}

Figure 5 shows an outbound NP pass of the northern polar cap, thus showing the reverse sequence of Fig. 3. This time, however, the IMF is directed steadily northward after 08:20 UT. Shortly afterwards $V_{s}$ becomes positive, i.e. the convection is now sunward, as expected over the central polar cap for strongly northward IMF (e.g. Reiff and Burch, 1985) for reconnection poleward of the cusp. At about 10:00 UT Cluster enters the cusp, and encounters downward directed plasma jets, with velocities up to $400 \mathrm{~km} \mathrm{~s}^{-1}$, presumably emanating from the magnetopause equatorward of the reconnection site. Already when approaching the cusp, and even more so inside, $V_{s}$ shows large-amplitude fluctuations that are reasonably well correlated between $\mathrm{C} 1$ and $\mathrm{C} 3$ (the $\mathrm{C} 2$ data stop earlier as a result of operational restrictions).

\footnotetext{
${ }^{2}$ in publication by Eriksson, A. I., et al. (see footnote on page 4)

${ }^{3}$ by Quinn, J. M., et al.: Convection Dropouts in the Polar Cap, in preparation.
}

\subsubsection{March 2001}

The final example (Fig. 6) shows an outbound NP pass over the northern polar cap towards the high-latitude magnetopause that is characterized by piecewise stable IMF, starting out almost exactly northward, becoming southward, and then switching back and forth a few more times. On average, the convection direction follows the IMF, but there are very large amplitude waves/fluctuations, commencing with entry into dense upflowing plasma. This case is subject of a separate publication ${ }^{4}$.

\subsection{Statistics}

In this section we present some statistical results concerning the dependence of the convection velocity and its fluctuations for all 20 cases listed in Table 1.

\subsubsection{Average convection}

In this section we look at the average convection as a function of the IMF. Figure 7 shows 10-min averages of convection velocities in the GSE (X, Z)-plane as a function of IMF $B_{Z}$, computed every two minutes for all 20 passes. As illustrated by Fig. 1 the spacecraft altitude varies substantially while passing over the polar cap. As the flux-tube cross section increases with altitude, so does the convection velocity. This explains why the convection velocity shown in Fig. 3, for example, becomes smaller with decreasing distance or increasing magnetic field strength. To remove the altitude effect, we do not show in Fig. 7 not the absolute velocities, but instead have scaled them to ionospheric altitudes of approximately $100 \mathrm{~km}$ by multiplication with $(B / 50000)^{1 / 2}$, where $B$ is the ambient magnetic field strength in $\mathrm{nT}$ and $50000 \mathrm{nT}$ is taken as the ionospheric field strength. This scaling explains why the magnitude of the velocities is now of the order of $1 \mathrm{~km} \mathrm{~s}^{-1}$. We denote the scaled convection velocity as $\tilde{V}_{s}$.

The figure shows the expected trend, in spite of the large scatter: for large negative IMF $B_{z}$ essentially all velocities are directed anti-sunward $\left(\tilde{V}_{s}<0\right)$, and the bulk of the velocities become smaller with increasing IMF $B_{z}$. The majority of the points show negative $\tilde{V}_{s}$ even for an IMF $B_{z}$ as large as $+5 \mathrm{nT}$. Some of the scatter certainly originates from uncertainties in the time-shift applied to the IMF data at times when the IMF orientation changes drastically. More importantly, however, is that reconnection poleward of the cusp does not necessarily imply sunward convection over the entire polar cap, because the existence of a cell that is closed within the polar cap implies that both sunward and antisunward convection exists (Cowley and Lockwood, 1992). It therefore depends on the spacecraft position which convection direction is actually observed. In fact, one of the crossings (21 March 2001, case 8 in Table 1) shows consistent anti-sunward convection even though the IMF points

\footnotetext{
${ }^{4}$ Puhl-Quinn, P. A., et al.: Cluster measures ULF waves over the polar cap, in preparation.
} 


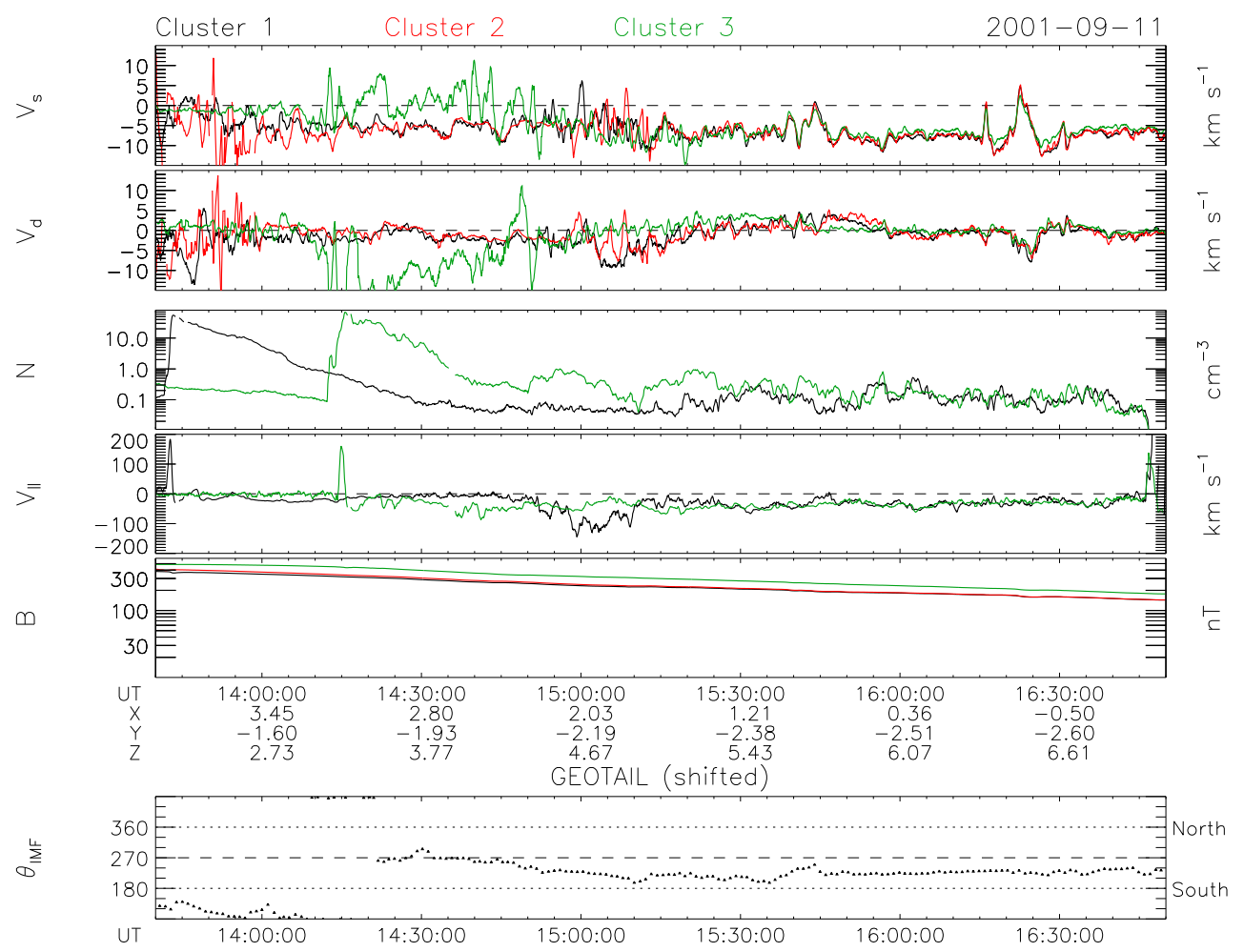

Fig. 4. Data for an outbound pass on 11 September 2001 in the same format as Fig. 3, except that EDI and FGM data are shown for C1, C2, and C3 (using the standard colors black, red and green, respectively), CIS-HIA data for C1 and C3 (not available on C2), and GEOTAIL data are used instead of ACE data for the IMF clock angle.

steadily northward, as the orbit maps to the dawnside of the central cell of the expected convection pattern, where for northward, duskward IMF the sense of convection is indeed anti-sunward.

We have also plotted the same data against the IMF clockangle. But since no asymmetry with regard to the IMF $B_{y}$ component (i.e. $90^{\circ}$ vs. $270^{\circ}$ clock-angle) was apparent, we do not show it here.

\subsubsection{Fluctuations}

In Sect. 3.2 we have seen that superimposed on the average convection there are often large-amplitude fluctuations. The level of fluctuations generally appeared to grow when approaching the cusp or magnetopause. To substantiate this finding, we show in Fig. 8 the variances of $\tilde{V}_{s}$, computed for the same 10-min intervals used to construct the data points in Fig. 7, as a function of the GSE-x spacecraft position relative to the position at the time of the closest approach to the cusp or magnetopause. Again, there is large scatter in the data, but the trend for increased variances when approaching the dayside polar cap boundary is apparent in the averages shown by the histogram for distances less than four Earth radii.

A similar though even weaker correlation is seen when plotting the variances vs. the plasma density. This probably simply reflects the fact that the density usually increases when approaching the cusp or magnetopause.

\subsubsection{Correlations}

In Sect. 3.2 we had noted the variablity of the correlation between the velocities measured by EDI on $\mathrm{C} 1, \mathrm{C} 2$, and $\mathrm{C} 3$. To further investigate these correlations we use 60 -s averages of the scaled velocities, $\tilde{V}_{s}$, to compute the cross-correlation coefficient between the 3 pairs of spacecraft for 60 min running averages, and plot them against the spacecraft separation distances (projected perpendicular to $\boldsymbol{B}$ ). Because of the noted expansion of the flux-tube cross section with altitude, a given spacecraft separation distance implies different scales when occurring at different spacecraft altitudes. To remove this effect, we have used the same scaling factor, $(B / 50000)^{1 / 2}$, already used to map the velocities, also for the spacecraft separation distance. The scaling factor ranges approximately from 0.03 to 0.1 , and the Cluster separations of a few $\mathrm{km}$ up to $10000 \mathrm{~km}$ are consequently mapped to very small scales in the ionosphere, down to a few hundred meters. Even the largest separations correspond to only a few hundred kilometers in the ionosphere. The resulting scatter plot is shown in Fig. 9a. It demonstrates that at small separations the correlation is almost always very good, while at large distances the correlation varies widely, ranging from very good to very poor.

To determine quantitatively which values for the correlation coefficients can be considered significantly different from zero we follow the standard statistical approach. First, 


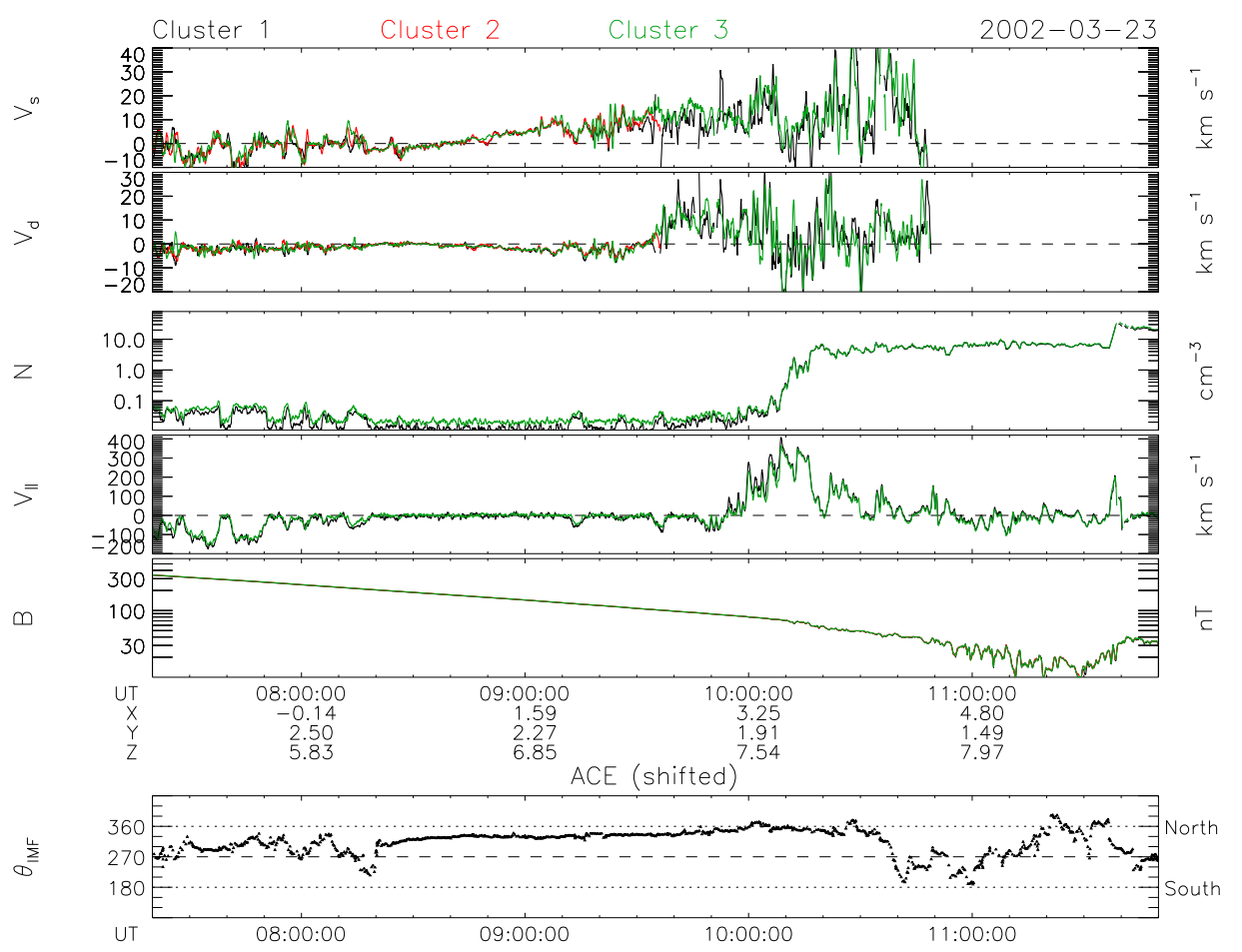

Fig. 5. Data for an outbound pass on 23 March 2002 over the northern polar cap towards the magnetopause, which is crossed near 11:40 UT. The average convection is mostly sunward, consistent with the mostly northward IMF. Note the downward directed high-speed plasma jets observed when entering the cusp near 10:00 UT. There are no useful EDI data after 10:50 UT because of the low and fluctuating magnetic field. EDI data on $\mathrm{C} 2$ stop even earlier because of operational restrictions.

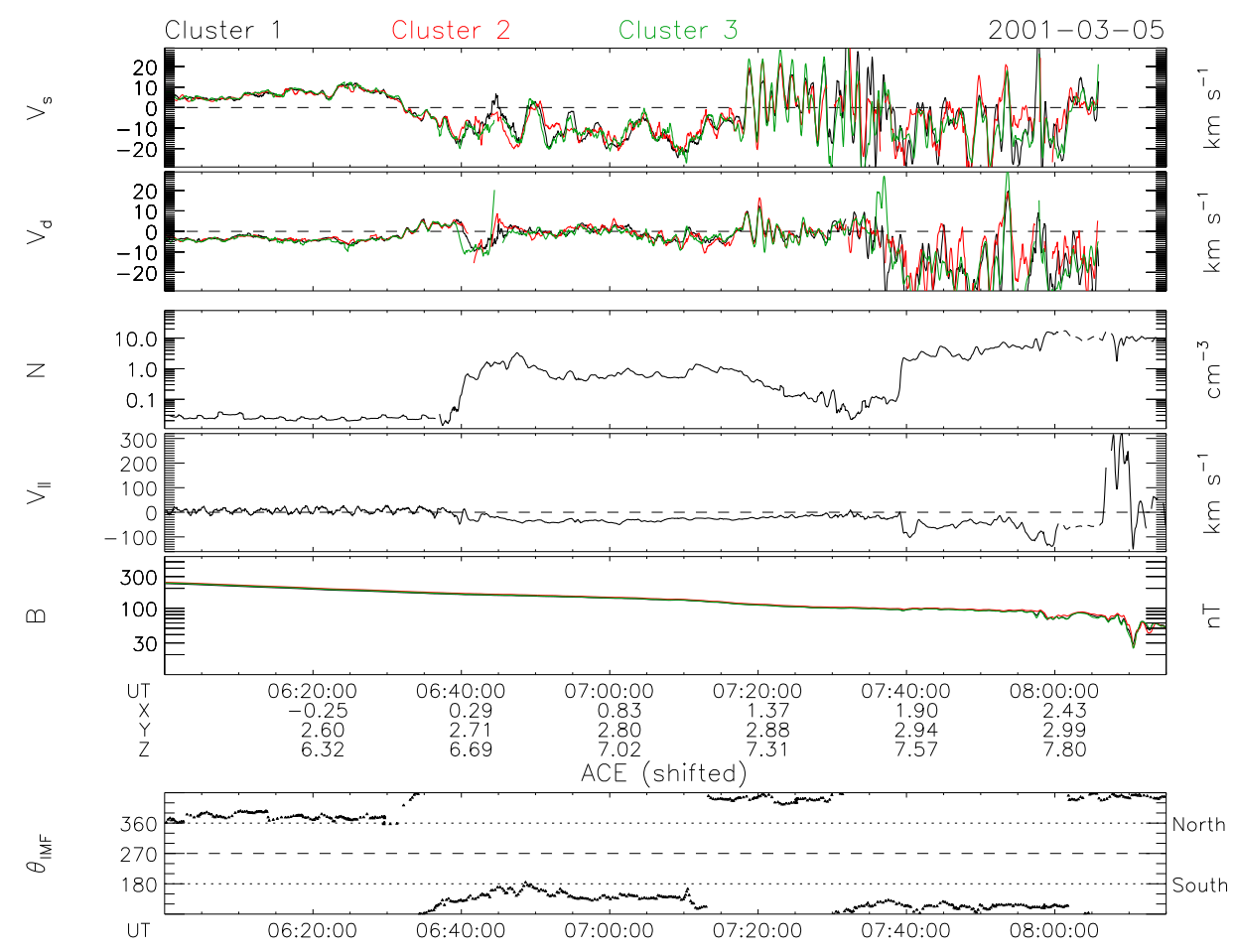

Fig. 6. Data for an outbound pass on 5 March 2001 in the same format as previous figures. The pass is characterized by transitions between piecewise stable IMF directions. The convection essentially follows the IMF, on average, but exhibits well correlated fluctuations with very large amplitudes when entering upward flowing plasma $\left(V_{\|}>0\right)$. The magnetopause is crossed near 08:10 UT. 


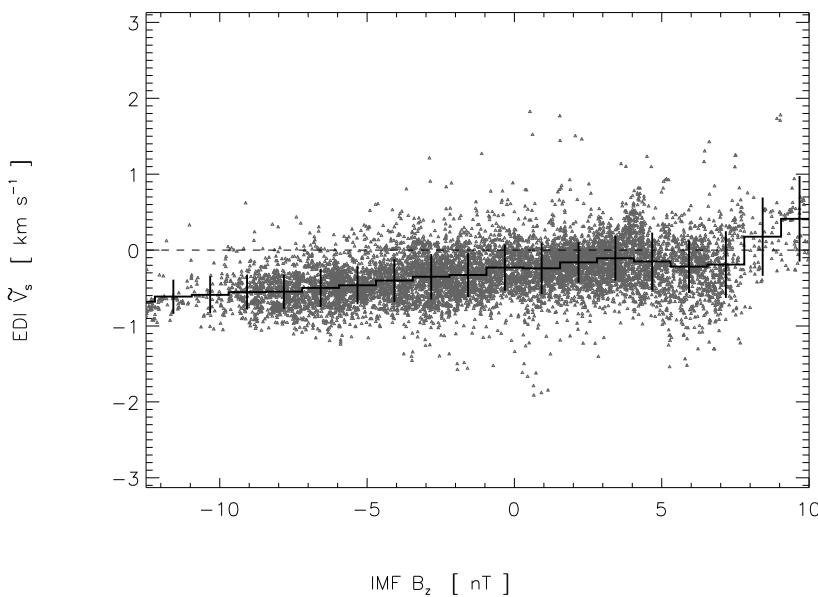

Fig. 7. Scaled 10-min averaged convection velocities $\tilde{V}_{S}$ vs. IMF $B_{z}$ for the 20 cases listed in Table 1. Overplotted in black are the average and standard deviations of the velocities.

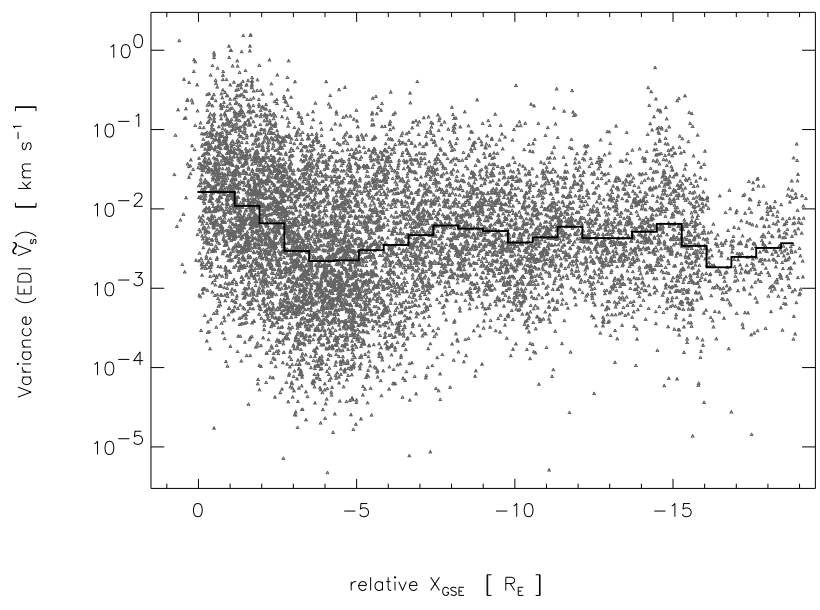

Fig. 8. Variances in $\tilde{V}_{s}$ for the same 10-min intervals as in Fig. 7, as a function of the distance along the GSE x-axis from the dayside polar cap boundary crossing.

we have confirmed that the distributions of the convection velocities $\tilde{V}_{s}$ are very close to Gaussian. Under the additional assumption (which we want to reject) that the true correlation coefficient is zero, the quantity

$t=\frac{r}{\sqrt{1-r^{2}}} \sqrt{N-2}$,

where $r$ is the calculated correlation coefficient and $N$ is the number of points used in the computation of the correlation coefficient, is $t$-distributed (Student's distribution) with $N$ 2 degrees of freedom. We consider the correlation coefficient to be significantly different from zero if the associated $t$-value lies in one of the two tails of the distribution, each of which holds $0.5 \%$ of the area of the integrated Student's distribution function. We have colored the correlation coefficients which pass this criterion in Fig. 9a blue. This analysis shows that insignificant correlation (colored black) exists mainly for separation distances above $20 \mathrm{~km}$.
For further analysis we have sorted the 60-min correlation coefficients into cases of northward and southward IMF, eliminating those 60-min intervals for which the IMF was not steadily pointing northward or southward. In Figs. 9b and c we have plotted the remaining correlation coefficients against the scaled spacecraft separation distance, and we have applied the same criterion as in Fig. 9a, to illustrate the question of significance of the correlation coefficients. These scatter plots clearly show that insignificant correlation exists mainly for northward IMF. In fact, for IMF $B_{z}>0$ significant (positive) correlation does not exist for mapped separations larger than approximately $200 \mathrm{~km}$. To quantify the different behaviour for northward and southward IMF we calculate a correlation scale length $\xi$ according to

$\xi=\frac{\sum_{i} \tilde{d}_{i \perp} r_{i}}{\sum_{i} r_{i}}$

where $\tilde{d}_{i \perp}$ are the scaled perpendicular spacecraft separations and $r_{i}$ are the associated correlation coefficients. The resulting values of $\xi$ are $31 \mathrm{~km}$ and $104 \mathrm{~km}$ for northward and southward IMF, respectively.

The smaller correlation scale lengths for northward IMF are consistent with models and observations reported in earlier publications. First, when the IMF changes from northward to southward, open magnetic flux is being added to the polar cap, causing the polar cap to grow until a quasi-steady equilibrium state is reached where the tail reconnection rate, on average, equals the reconnection rate at the dayside magnetopause (Cowley and Lockwood, 1992). A second contributing factor is that there is a tendency for more convection cells for northward IMF (e.g. Reiff and Burch, 1985; Cowley and Lockwood, 1992), compared to southward IMF, with more flow reversals along the dawn-dusk line. Furthermore, Maynard et al. (1998) reported the reconnection poleward of the cusp for northward IMF, that leads to lobe cells, to be patchy. Moreover, since the lobe cells for northward IMF are closed within the polar cap, one should, in principle, even be able to observe anticorrelation at larger spacecraft separation distances (if the separation is mainly in the dawn-dusk direction). The trend in Fig. $9 \mathrm{~b}$ for large separation distances is consistent with this prediction.

\section{Summary and conclusions}

The findings presented in the previous sections may be summarized as follows:

1. On average, the convection velocity shows the expected dependence on IMF- $B_{z}$ (or clock-angle). However, there is large scatter, even though the velocities were already averaged over $10 \mathrm{~min}$. While some of the scatter is attributable to uncertainties in the propagation delay of the upstream monitor data, it is also clear that even for stable IMF conditions there are large variations in the 10-min averaged convection velocities that obscure 

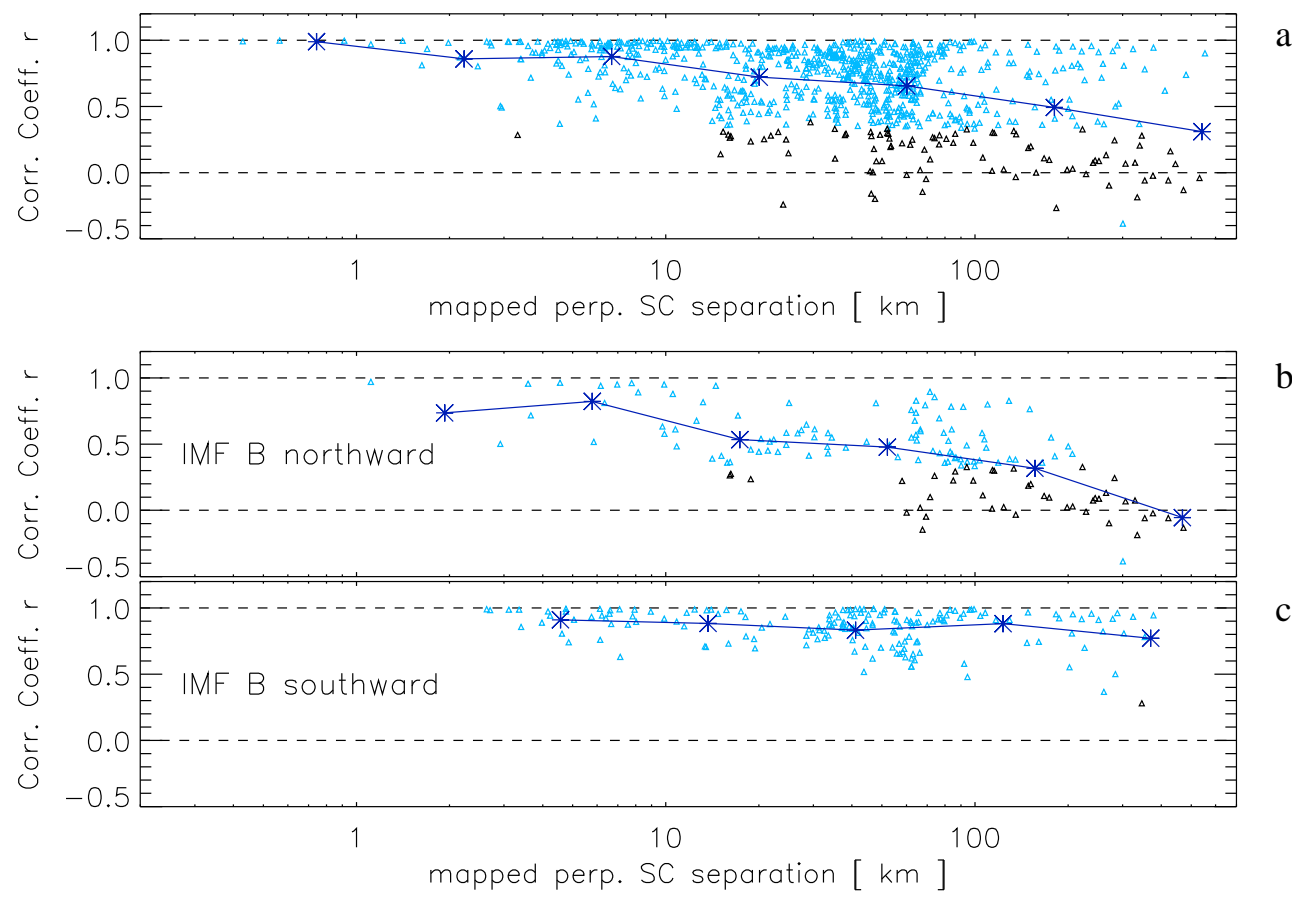

Fig. 9. (a) Correlation coefficients of 1-h intervals of 60-s smoothed $\tilde{V}_{s}$ vs. mapped spacecraft separation distances transverse to the magnetic field; correlation coefficients above the significance threshold are colored blue, the others black; (b) and (c) same as (a), except the subset of cases with steady IMF have been separated into IMF northward and southward, respectively. The asterisks interconnected by straight lines show the average trend.

the average behavior. For northward IMF, the convection is not expected to be uniformly sunward over the entire polar cap, but contains regions with anti-sunward convection, the detailed distribution depending on IMF$B_{y}$. This effect contributes to the large scatter seen for northward IMF.

When considering the dependence of in-situ convection measurements one must also not forget that convection is the sum of two intrinsically time-dependent contributions. One is driven by coupling processes at the magnetopause, primarily by magnetic reconnection, and determined by concurrent IMF/SW conditions. The other is driven by tail-processes and related to past history of the IMF/SW and the magnetosphere (Cowley and Lockwood, 1992). As our cases extend well into the nightside polar cap it is quite likely that some of the variations we observe are due to magnetotail processes. The dropouts noted in conjunction with the 11 September 2001 event (Sect. 3.2.2) are a particularly relevant case at hand.

To make statements about the (co-)existence of the various kinds of convection cells (merging, lobe, viscous) would require one to distinguish the data according to the signs of IMF $B_{y}$ and IMF $B_{z}$, as well as the spacecraft position along the dawn-dusk line. This would require more than the limited number of cases available for this study. In particular, we cannot contribute to the question of the existence of lobe-type cells for southward IMF, as they have been reported, e.g. by Burch et al. (1985) and Eriksson et al. (2002).

2. Perhaps the most striking feature in the EDI data is the occurrence of large amplitude fluctuations that are superimposed on the average behavior. From inspection of the individual cases, and confirmed by the statistical analysis of all 20 cases, it is evident that there is one type of fluctuation that grow when approaching the cusp/magnetopause boundary and its occurrence is thus not a temporal phenomenon. But there are other cases when large variances seem to occur at random.

One contributing factor to the large fluctuations observed in the vicinity of the boundaries, especially the magnetopause, is the motion of the boundaries themselves. We have cases of multiple magnetopause crossings where the sign and magnitude of the EDI velocities match the inward and outward motion of the magnetopause inferred from four spacecraft timing analysis.

3. The unique contribution of Cluster is the ability to provide multi-point measurements, thus allowing the inference of spatial scales. From correlations of the convection velocities from the three spacecraft where EDI is operative, it appears that the scales, when mapping the spacecraft separation distances down to ionospheric altitudes, are always larger than $1 \mathrm{~km}$ and sometimes, but 
not always larger than a few hundred $\mathrm{km}$. Splitting the data into cases of pure northward and southward IMF shows that the large scales of a few hundred km exist only for southward IMF, whereas for northward IMF poor correlation occurs already at separation distances of only a few tens of $\mathrm{km}$. These results are in qualitative agreement with models and observations reported by Cowley and Lockwood (1992), Reiff and Burch (1985) and Maynard et al. (1998). While it seems hardly surprising that scales are larger than $1 \mathrm{~km}$, poor correlation at only a few tens of $\mathrm{km}$ separation over the polar cap is surprising and is below the resolution achieved by the SuperDARN radars in their standard scan mode.

Acknowledgement. The authors would like to thank M. Chutter and T. Leistner for data processing support, and the ACE SWEPAM and MAG instrument teams, as well as the GEOTAIL CPI and MGF instrument teams and the CDAWeb for the Solar Wind/IMF data. This work was supported by DLR through grants FKZ:50OC89043 and FKZ:50OC9705, and by NASA through grants NNG04GA46G and NAG5-9960.

Topical Editor T. Pulkkinen thanks S. W. H. Cowley and another referee for their help in evaluating this paper.

\section{References}

Balogh, A., Dunlop, M. W., Cowley, S. W. H., Southwood, D. J., Thomlinson, J. G., Glassmeier, K. H., Musmann, G., Luhr, H., Buchert, S., Acuna, M. H., Fairfield, D. H., Slavin, J. A., Riedler, W., Schwingenschuh, K., and Kivelson, M. G.: The Cluster Magnetic Field Investigation, Space Sci. Rev., 79, 65-91, 1997.

Burch, J. L., Reiff, P. H., Menietti, J. D., Winningham, J. D., Heelis, R. A., Hanson, W. B., Shawhan, S. D., Shelley, E. G., Sugiura, M., and Weimer, D. R.: IMF By-dependent plasma flow and Birkeland currents in the dayside magnetosphere, I. - Dynamics Explorer observations, J. Geophys. Res., 90, 1577-1593, 1985.

Cowley, S. W. H. and Lockwood, M.: Excitation and decay of solar wind-driven flows in the magnetosphere-ionosphere system, Ann. Geophys., 10, 103-115, 1992.

Eriksson, S., Bonnell, J. W., Blomberg, L. G., Ergun, R. E., Marklund, G. T., and Carlson, C. W.: Lobe cell convection and fieldaligned currents poleward of the region 1 current system, J. Geophys. Res.-Space, 107(A8), 10.1029/2001JA005041, 2002.

Gustafsson, G., Bostrom, R., Holback, B., Holmgren, G., Lundgren, A., Stasiewicz, K., Ahlen, L., Mozer, F. S., Pankow, D., Harvey, P., Berg, P., Ulrich, R., Pedersen, A., Schmidt, R., Butler, A., Fransen, A. W. C., Klinge, D., Thomsen, M., Falthammar, C.G., Lindqvist, P.-A., Christenson, S., Holtet, J., Lybekk, B., Sten, T. A., Tanskanen, P., Lappalainen, K., and Wygant, J.: The Electric Field and Wave Experiment for the Cluster Mission, Space Sci. Rev., 79, 137-156, 1997.

Lockwood, M. and Moen, J.: Reconfiguration and closure of lobe flux by reconnection during northward IMF: possible evidence for signatures in cusp/cleft auroral emissions, Ann. Geophys., 17, 996-1011, 1999.

Maynard, N. C., Burke, W. J., Weimer, D. R., Mozer, F. S., Scudder, J. D., Russell, C. T., Peterson, W. K., and Lepping, R. P.: Polar observations of convection with northward interplanetary magnetic field at dayside high latitudes, J. Geophys. Res., 103, 29-46, 1998.
Paschmann, G., Melzner, F., Frenzel, R., Vaith, H., Parigger, P., Pagel, U., Bauer, O. H., Haerendel, G., Baumjohann, W., Scopke, N., Torbert, R. B., Briggs, B., Chan, J., Lynch, K., Morey, K., Quinn, J. M., Simpson, D., Young, C., McIlwain, C. E., Fillius, W., Kerr, S. S., Mahieu, R., and Whipple, E. C.: The Electron Drift Instrument for Cluster, Space Sci. Rev., 79, 233-269, 1997.

Paschmann, G., Quinn, J. M., Torbert, R. B., Vaith, H., McIlwain, C. E., Haerendel, G., Bauer, O. H., Bauer, T., Baumjohann, W. Fillius, W., Förster, M., Frey, S., Georgescu, E., Kerr, S. S., Kletzing, C. A., Matsui, H., Puhl-Quinn, P., and Whipple, E. C.: The Electron Drift Instrument on Cluster: overview of first results, Ann. Geophys., 19, 1273-1288, 2001.

Quinn, J. M., Paschmann, G., Torbert, R. B., Vaith, H., McIlwain, C. E., Haerendel, G., Bauer, O., Bauer, T. M., Baumjohann, W., Fillius, W., Foerster, M., Frey, S., Georgescu, E., Kerr, S. S., Kletzing, C. A., Matsui, H., Puhl-Quinn, P. A., and Whipple, E. C.: Cluster EDI convection measurements across the highlatitude plasma sheet boundary at midnight, Ann. Geophys., 19, 1669-1681, 2001.

Reiff, P. H. and Burch, J. L.: IMF B(y)-dependent plasma flow and Birkeland currents in the dayside magnetosphere, II. - A global model for northward and southward IMF, J. Geophys. Res., 90, 1595-1609, 1985.

Rème, H., Aoustin, C., Bosqued, J. M., Dandouras, J., Lavraud, B., Sauvaud, J. A., Barthe, A., Bouyssou, J., Camus, T., Coeur-Joly, O., Cros, A., Cuvilo, J., Ducay, F., Garbarowitz, Y., Medale, J. L., Penou, E., Perrier, H., Romefort, D., Rouzaud, J., Vallat, C., Alcaydé, D., Jacquey, C., Mazelle, C., D‘Uston, C., Möbius, E., Kistler, L. M., Crocker, K., Granoff, M., Mouikis, C., Popecki, M., Vosbury, M., Klecker, B., Hovestadt, D., Kucharek, H., Kuenneth, E., Paschmann, G., Scholer, M., Sckopke (†), N., Seidenschwang, E., Carlson, C. W., Curtis, D. W., Ingraham, C., Lin, R. P., McFadden, J. P., Parks, G. K., Phan, T., Formisano, V., Amata, E., Bavassano-Cattaneo, M. B., Baldetti, P., Bruno, R., Chionchio, G., di Lellis, A., Marcucci, M. F., Pallocchia, G., Korth, A., Daly, P. W., Graeve, B., Rosenbauer, H., Vasyliunas, V., McCarthy, M., Wilber, M., Eliasson, L., Lundin, R., Olsen, S., Shelley, E. G., Fuselier, S., Ghielmetti, A. G., Lennartsson, W., Escoubet, C. P., Balsiger, H., Friedel, R., Cao, J.-B., Kovrazhkin, R. A., Papamastorakis, I., Pellat, R., Scudder, J., and Sonnerup, B.: First multispacecraft ion measurements in and near the Earth's magnetosphere with the identical Cluster ion spectrometry (CIS) experiment, Ann. Geophys., 19, 1303-1354, 2001.

Ruohoniemi, J. M. and Baker, K. B.: Large-scale imaging of highlatitude convection with Super Dual Auroral Radar Network HF radar observations, J. Geophys. Res., 103, 20 797-20 812, 1998.

Tsyganenko, N. A.: A model of the near magnetosphere with a dawn-dusk asymmetry, 1. Mathematical structure, J. Geophys. Res. (Space Physics), 107(A8), 10.1029/2001JA000219, 2002.

Weimer, D. R.: A flexible, IMF-dependent model of high-latitude electric potentials having "space weather" applications, Geophys. Res. Lett., 23, 2549-2552, 1996. 